\title{
DETECTING AND DISCARDING OF SHADOWS IN IMAGE USING GEOMETRIC CONTOURS AND REGION BASED SEGMENTATION USING THRESHOLDING APPROACH
}

Shanthi. $S^{* 1}$, Vinothini. K. R ${ }^{2}$, R. Manikandan ${ }^{3}$

*1, 2, 3 Department of Electronics and Communication Engineering, AVC College of Engineering, Annai College of Engineering \& Technology, INDIA

*Correspondence Author: shanthis256@gmail.com

\section{Abstract:}

Shadow detection and removal is an important task when dealing with color outdoor images. Shadows are generated by a local and relative absence of light. Most shadow detection and segmentation methods are based on image analysis. However, some factors will affect the detection result due to the complexity of the circumstances. In this paper, a new algorithm for shadow detection and isolation of buildings in high-resolution panchromatic satellite imagery is proposed. This algorithm is based on tailoring the traditional model of the geometric active contours such that the new model of the contours is systematically biased toward segmenting the shadow and the dark regions in the image. Feature extraction a type of dimensionality reduction that efficiently represents interesting parts of an image as a compact feature vector. This approach is useful when image sizes are large and a reduced feature representation is required to quickly complete tasks such as image matching and retrieval.

Keywords:

Building Detection, Geometric Active Contours, Image Segmentation, Shadow Detection, Feature Extraction.

Cite This Article: Shanthi. S, Vinothini. K. R, R. Manikandan, "Detecting and Discarding of Shadows in Image Using Geometric Contours and Region Based Segmentation Using Thresholding Approach." International Journal of Research - Granthaalayah, Vol. 3, No. 2(2015): 13-19. DOI: https://doi.org/10.29121/granthaalayah.v3.i2.2015.3036.

\section{INTRODUCTION}

Satellite and aerial imaging is a common method to obtain information about objects on the Earth's surface. Object and target detection is of great interest for many applications, including rescue operations and defense applications. Recently, extension of object detection to man-made structure (e.g., buildings) detection and recognition in aerial images has attracted attention. The ability to effectively detect structures helps in understanding the scene contents of the image and maybe used for content-based retrieval in databases and in other applications such as residential development planning, damage evaluation, and military target detection. Shadow is a crucial cue for detecting the existence of the buildings and other man-made structures in the overhead images. Shadow of buildings is isolated and then is employed for detection by integrating and fusing the geometry of the shadow area with the potential geometry of the building or other elevated man- 


\section{INTERNATIONAL JOURNAL of RESEARCH -GRANTHAALAYAH

made structures. However, there is a general lack of shadow detection methods for panchromatic imagery and particularly for shadow of man-made buildings in these images in literature.

The problem of shadowing is particularly significant in high-resolution (approximately $1 \mathrm{~m}$ ) satellite imaging (HRSI). The equatorial crossing time of 1030 of the Ikonos satellite was chosen to ensure stable imaging conditions (the atmosphere is generally clearer in the morning than later in the day). This, however, means that the solar elevation will never be high, irrespective of latitude and season. The effects of shadowing can be reduced slightly by increasing the look angle of the sensor (basically looking at a region on the ground where the local solar time is slightly later)but this in turn increases the occlusion of features in the image. The effects of shadowing are compounded in regions where there are dramatic changes in surface elevation, namely in urban areas. Even the smaller buildings are casting shadows that are obscuring details on the surrounding streets. It is somewhat ironic that the features that high resolution satellite sensors have been designed to image are those features which are most affected by shadowing. In comparison, largescale features, which are more appropriately imaged by lower resolution sensors such as regional vegetation patterns, consist of fewer dramatic changes in elevation, and hence, are less likely to be affected by their own shadows.

\section{SYSTEM ARCHITECTURE}

\subsection{PROPOSED SYSTEM}

The proposed method is based on the geometric active-contour model. The geometric activecontour model is based on image segmentation. The contour model is represented by the zero-level set of the higher dimensional function in the level-set framework. These functions are able to detect the boundary of regions based on the homogeneity of local features such as the intensity without depending on the edges of the region as motivation force. Figure 1. Shows block diagram of proposed method.

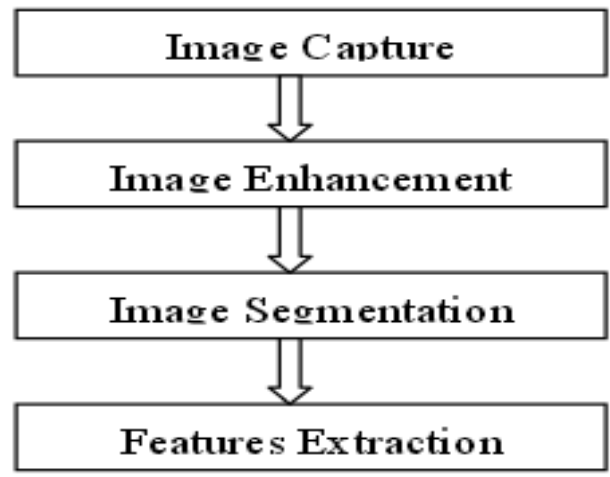

Figure 1: Block diagram of the proposed method. 


\section{INTERNATIONAL JOURNAL of RESEARCH -GRANTHAALAYAH

\subsection{FLOW DIAGRAM}

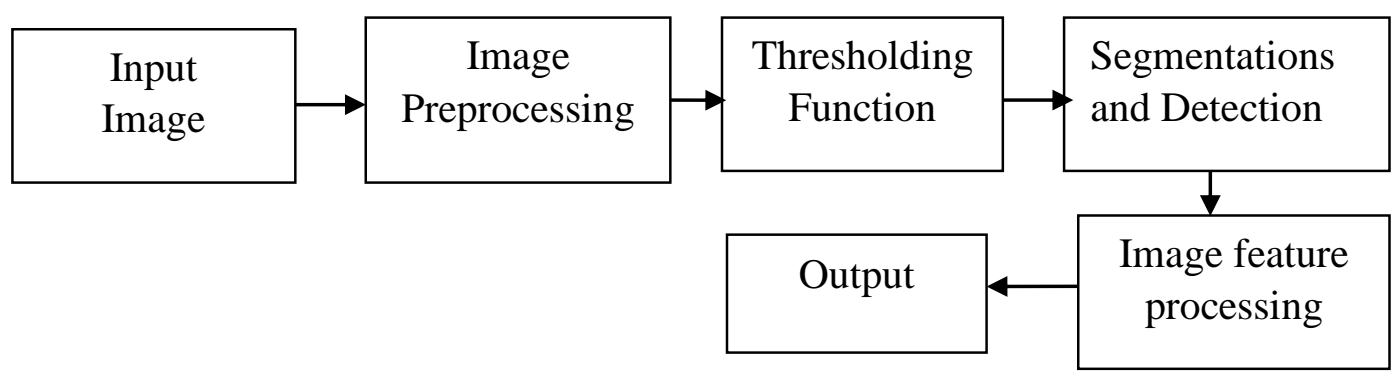

Figure 2: Flow diagram

\section{OVERALL ARCHITECTURE}

\subsection{MODULES DESCRIPTION}

\subsubsection{THRESHOLDING FUNCTION}

The simplest thresholding methods replace each pixel in an image with a black pixel if the image intensity $I_{i, j}$ is less than some fixed constant $\mathrm{T}$ (that is, $I_{i_{i} j}<T$ ), or a white pixel if the image intensity is greater than that constant. Here, Clustering-based methods is used, where the graylevel samples are clustered in two parts as background and foreground (object), or alternately are modeled as a mixture of two Gaussians.

\subsubsection{IMAGE SEGMENTATION}

The proposed method is based on the geometric active-contour model. The geometric activecontour model is based on image segmentation. The contour model is represented by the zero-level set of the higher dimensional function in the level-set framework. These functions are able to detect the boundary of regions based on the homogeneity of local features such as the intensity without depending on the edges of the region as motivation force. Segmentation using active contours model (Snakes) was introduced. The idea behind active contours, or deformable models, for image segmentation is quite simple. The user specifies an initial guess for the contour, which is then moved by image driven forces to the boundaries of the desired objects. In such models, two types of forces are considered - the internal forces, defined within the curve, are designed to keep the model smooth during the deformation process, while the external forces, which are computed from the underlying image data, are defined to move the model toward an object boundary or other desired features within the image. One way of describing this curve is by using an explicit parametric form, which is the approach used in snakes. 


\section{INTERNATIONAL JOURNAL of RESEARCH -GRANTHAALAYAH

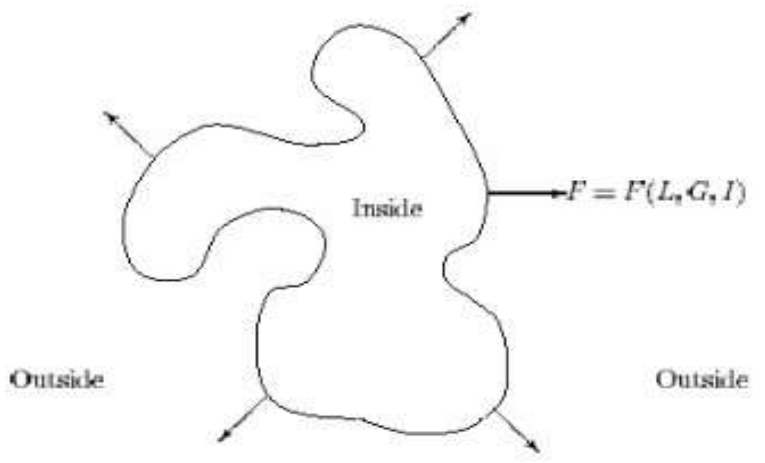

Figure 3: Snake approach

\subsubsection{FEATURE EXTRACTION}

The feature is defined as a function of one or more measurements, each of which specifies some quantifiable property of an object, and is computed such that it quantifies some significant characteristics of the object. We classify the various features currently employed as follows:

- General features: Application independent features such as color, texture, and shape. According to the abstraction level, they can be further divided into:

1. Pixel-level features: Features calculated at each pixel, e.g. color, location.

2. Local features: Features calculated over the results of subdivision of the image band on image segmentation or edge detection.

3. Global features: Features calculated over the entire image or just regular subarea of an image.

- Domain-specific features: Application dependent features such as human faces, fingerprints, and conceptual features. These features are often a synthesis of lowlevel features for a specific domain.

On the other hand, all features can be coarsely classified into low-level features and highlevel features. Low-level features can be extracted directed from the original images, whereas high-level feature extraction must be based on low level features

\section{RESULTS}

The images used in this paper are publicly provided by the U.S. Geological Survey, and each image consists of four bands (RGBIR) with a resolution of one pixel per meter in both directions. For these color images, we created the corresponding panchromatic images for our application. The images contain various objects, including vegetation, buildings, towers, water bodies, roads, and other man-made objects such as cars and trucks. 


\section{INTERNATIONAL JOURNAL of RESEARCH -GRANTHAALAYAH

Choose the scenes such that they contain various scenarios for buildings contents such as isolated buildings, connected buildings, small building, and large buildings. In addition, the shadow contents in the input images vary in the shape and the size such that the shadows might be represented by a few pixels to a large region. In addition, the shadow region can either be isolated or connected to vegetation and/or water bodies. Figure 4. Shows input image for proposed algorithm and Figure 6. Shows the shadow region segmentation image.

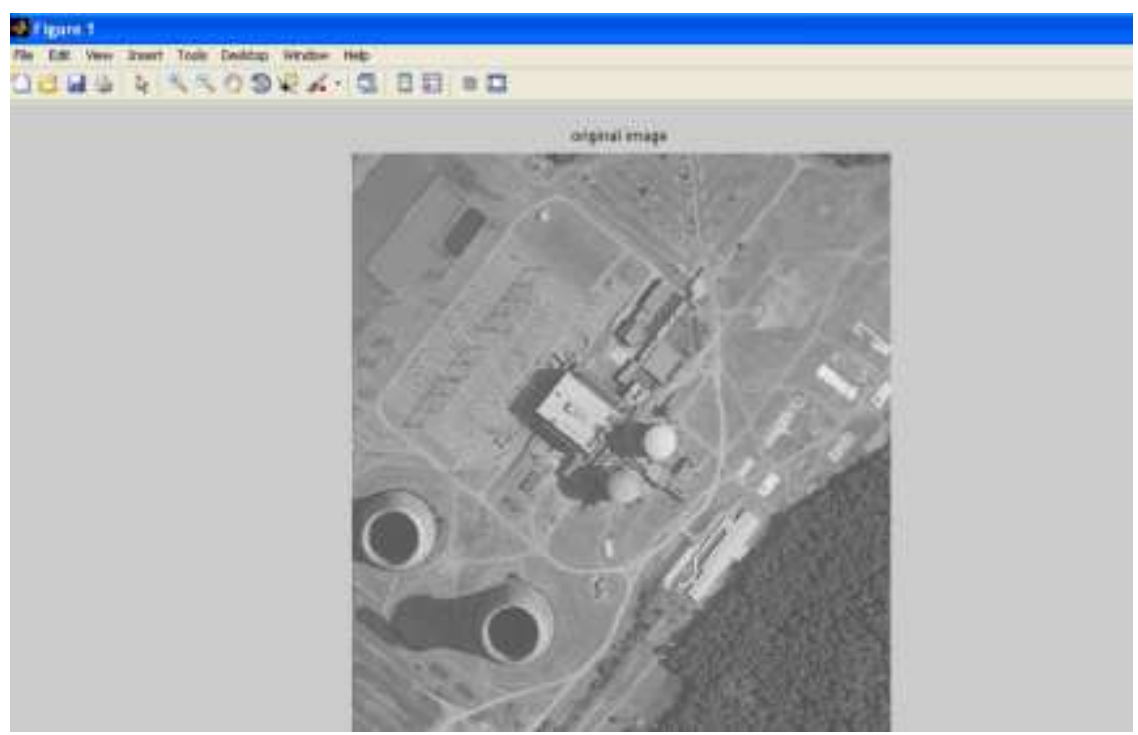

Figure 4: input image

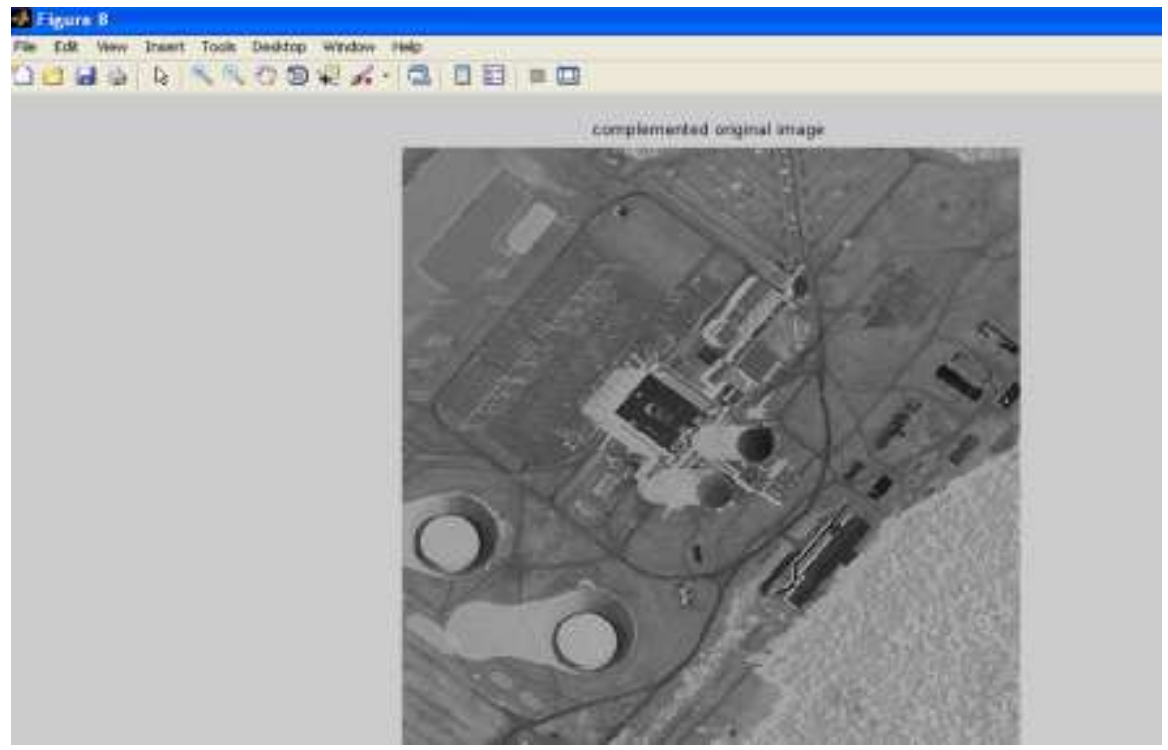

Figure 5: complemented input image 


\section{INTERNATIONAL JOURNAL of RESEARCH -GRANTHAALAYAH}

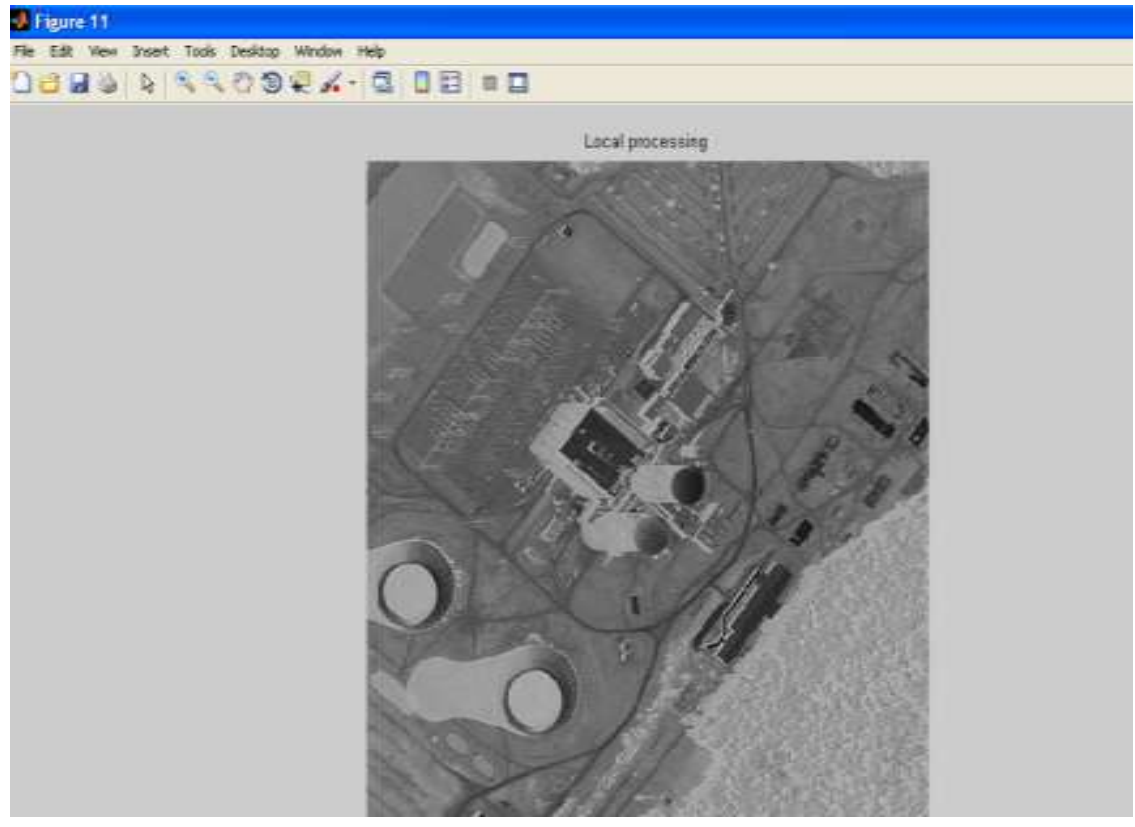

Figure 6: Shadow region segmented image

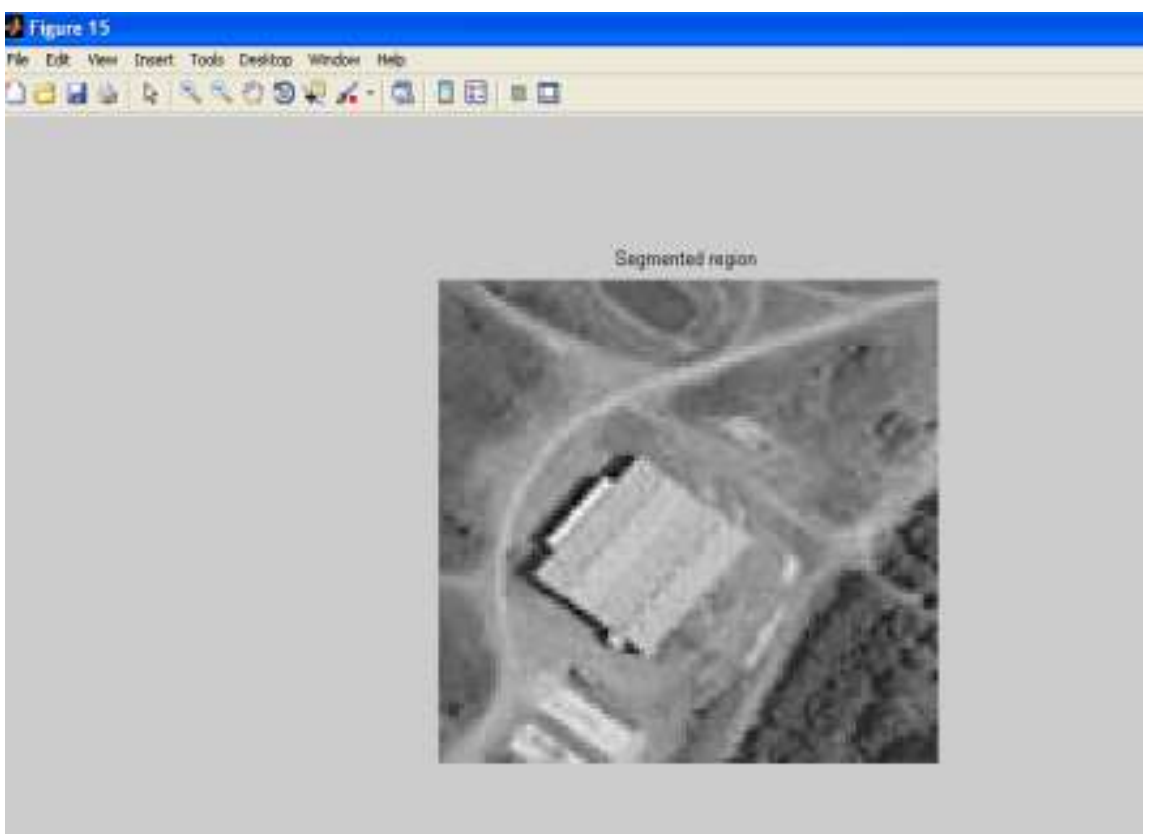

Figure 7: Building segmentation image 


\section{INTERNATIONAL JOURNAL of RESEARCH -GRANTHAALAYAH \\ A knowledge Repository}

Science

\section{CONCLUSION}

Shadows are detection and extraction from high-resolution panchromatic satellite images. The proposed technique segments the shadow of man-made structures such as buildings by using the gray-level satellite image without using the color information. The algorithm is based on employing an improved geometric active-contour model to handle a rather difficult problem of shadow detection in satellite imagery. Our proposed model of the geometric active contours systematically favors the shadow and the similar dark regions in the input image. Further processing steps are introduced to isolate the shadow from clutter such as vegetation and water bodies. Both qualitative and quantitative experimental results using real images show that the proposed algorithm outperforms a comparable algorithm for shadow and man-made structure segmentation.

\section{REFERENCES}

[1] Adeline .K. R. M, M. Chen, X. Briottet, S. K. Pang, and N. Paparoditis, "Shadow detection in very high spatial resolution aerial images: A comparative study," ISPRS J. Photogramm Remote Sens., vol. 80, pp. 21-38, Jun. 2013.

[2] Al-Najdawi.N, H. E. Bez, J. Singhai, and E. A. Edirisinghe, "A survey of cast sshadow detection algorithms," Pattern Recognit. Lett., vol. 33, no. 6, pp. 752-764, Apr. 2012.

[3] Arbel.E and H. Hel-Or, "Shadow removal using intensity surfaces and texture anchor points," IEEE Trans. Pattern Anal. Mach. Intell., vol. 33,no. 6, pp. 1202-1216, Jun. 2011.

[4] Chung. K. L, Y. R. Lin, and Y. H. Huang, "Efficient shadow detection of color aerial images based on successive thresholding scheme," IEEE Trans. Geosci. Remote Sens., vol. 47, no. 2, pp. 671-682, Feb. 2009.

[5] Guo.R, Q. Dai, and D. Hoiem, "Single-image shadow detection and removal using paired regions," in Proc. CVPR, Jun. 20-25, 2011, pp. 2033-2040.

[6] Huang.X and L. Zhang, "Morphological building/shadow index for building extraction from high-resolution imagery over urban areas," IEEE J.Sel. Topics. Appl. Earth Observ. Remote Sens., vol. 5, no. 1, pp. 161-172,Feb. 2012.

[7] Liu.Z, K. Huang, and T. Tan, "Cast shadow removal in a hierarchical manner using MRF," IEEE Trans. Circuits Syst. Video Tech., vol. 22, no. 1,pp. 56-66, Jan. 2012. 